\title{
Spinal Metastasis from Struma Ovarii: Case Report and Review of the Literature
}

\author{
Kazuyoshi Kobayashi ${ }^{1}$, Shiro Imagama ${ }^{1}$, Shin Tsunekawa $^{2}$, Kaori Hosokawa ${ }^{2}$, \\ Minemori Watanabe ${ }^{3}$, Zenya Ito ${ }^{1}$, Kei Ando ${ }^{1}$, Naoki Ishiguro ${ }^{1}$ \\ ${ }^{I}$ Department of Orthopaedic Surgery, Nagoya University Graduate School of Medicine, Nagoya, Japan \\ ${ }^{2}$ Department of Endocrinology, Nagoya University Graduate School of Medicine, Nagoya, Japan \\ ${ }^{3}$ Department of Endocrinology, Okazaki City Hospital, Okazaki, Japan
}

\begin{abstract}
Struma ovarii is a rare tumor that is defined as an ovarian teratoma with a thyroid tissue component exceeding $50 \%$. Most of these tumors are benign, with malignant struma ovarii occurring in $<1 \%$ of patients. Here, we describe the case of a 49-year-old female patient with malignant struma ovarii who developed thoracic spine metastasis. She had undergone an oophorectomy and was diagnosed with struma ovarii 10 years previously. She had remained recurrence-free thereafter. At 49 years of age, she developed low back pain and was admitted to our hospital for evaluation of a spinal tumor at the Th7 level. An emergency bone biopsy led to a diagnosis of metastasis from malignant struma ovarii. External beam radiotherapy inhibited further tumor growth and there was no resulting muscle weakness. This is the first report of spinal metastasis occurring 10 years after resection of struma ovarii, indicating the need for long-term follow-up.
\end{abstract}

Keywords: Neoplasms; Struma ovarii; Spinal neoplasms; Thyroid; Recurrence

\section{Introduction}

Struma ovarii is a rare form of ovarian teratoma comprising $>50 \%$ of thyroid tissue [1] that represents about $1 \%$ of all ovarian teratomas [2]. It is histologically, physiologically, and pharmacologically identical to normal thyroid tissue [2], and is usually benign and only rarely malignant [3]. Distant metastases from malignant struma ovarii are also rare and occur in about $5 \%$ of cases [1]. These metastases can spread via the lymphatic system to pelvic and paraortic lymph nodes; via direct spread to the omentum, peritoneal cavity, and contralateral ovary; and via hematologic transmission to the bone, brain, lung, contralateral ovary, and liver [1]. The common sites of metastasis for struma ovarii are the peritoneum, mesentery, and omentum followed by (in decreasing order of frequency) the liver, contralateral ovary, bone, and lung [4]. Bone is not a common site for metastasis and there are some reported cases of struma ovarii with bony metastasis in the English literature [2-7]. But, only 5 cases of spinal metastasis from malignant struma ovarii have been described [2,5-7].

We experienced a case of a 49-year-old woman who developed thoracic vertebral bone metastasis 10 years after resection of struma ovarii. The 10-year period before recurrence is the longest among all published cases. Here, we present this rare case with a literature review.

Received Mar 11, 2014; Revised Apr 24, 2014; Accepted Apr 25, 2014

Corresponding author: Shiro Imagama

Department of Orthopaedic Surgery, Nagoya University Graduate School of Medicine,

65, Tsurumai-cho, Showa-ku, Nagoya 466-8560, Japan

Tel: +81-52-741-2111, Fax: +81-52-744-2260, E-mail: imagama@med.nagoya-u.ac.jp 


\section{Case Report}

At 39-years-of-age, a woman was incidentally diagnosed with struma ovarii after a surgical procedure for ovarian torsion. Pathological examination revealed proliferation of follicular epithelium and the presence of thyroid tissue (Fig. 1A, B). The MIB-1 index (Ki67) was 1\%-2\% (Fig. 1C). There was no recurrence of the tumor after surgery. Presently, 10 years later, she experienced low back pain. Magnetic resonance imaging (MRI) and computed tomography (CT) performed at a local hospital revealed a large tumor surrounding the spinal cord accompanied by osteolysis at the Th7 level (Fig. 2), and she consulted with our hospital. Despite severe spinal cord compression, there was no lower limb muscle weakness. We performed an emergency bone biopsy and detected hyperthyroglobinemia in tissues collected via a puncture needle from thoracic lesions. The serum thyroglobulin level was $38,242 \mu \mathrm{g} / \mathrm{L}$ (reference range, $2-32 \mu \mathrm{g} / \mathrm{L}$ ). Pathological examination of the $\mathrm{Th} 7$ lamina bone revealed a metastatic follicular thyroid carcinoma. Atypical cells with nuclear swelling formed a follicle based on histological analysis (Fig. 3). Histological findings were consistent with metastasis from malignant struma ovarii.

Following thyroid examination and whole-body positron emission tomography, we excluded the possibility of primary thyroid malignancy and detected metastasis to the ilium (Fig. 4). There was no ovarian tumor recurrence or other visible metastases. The final diagnosis was metastasis from malignant struma ovarii.

Because thoracic spinal cord compression was significant, we initially considered tumor resection. However, the patient and her family decided against this procedure based on the association of intralesional resection of a tumor surrounding the spinal cord with risks of massive bleeding and postoperative paralysis. She eventually underwent external beam radiotherapy at a dose of $37.5 \mathrm{~Gy}$ for the spinal lesion. She continued to lie in bed until end of radiotherapy course, then became ambulatory using a rigid Th7 and lumbar brace. During a rehabilitation period of several weeks, she attained stable walking with rigid
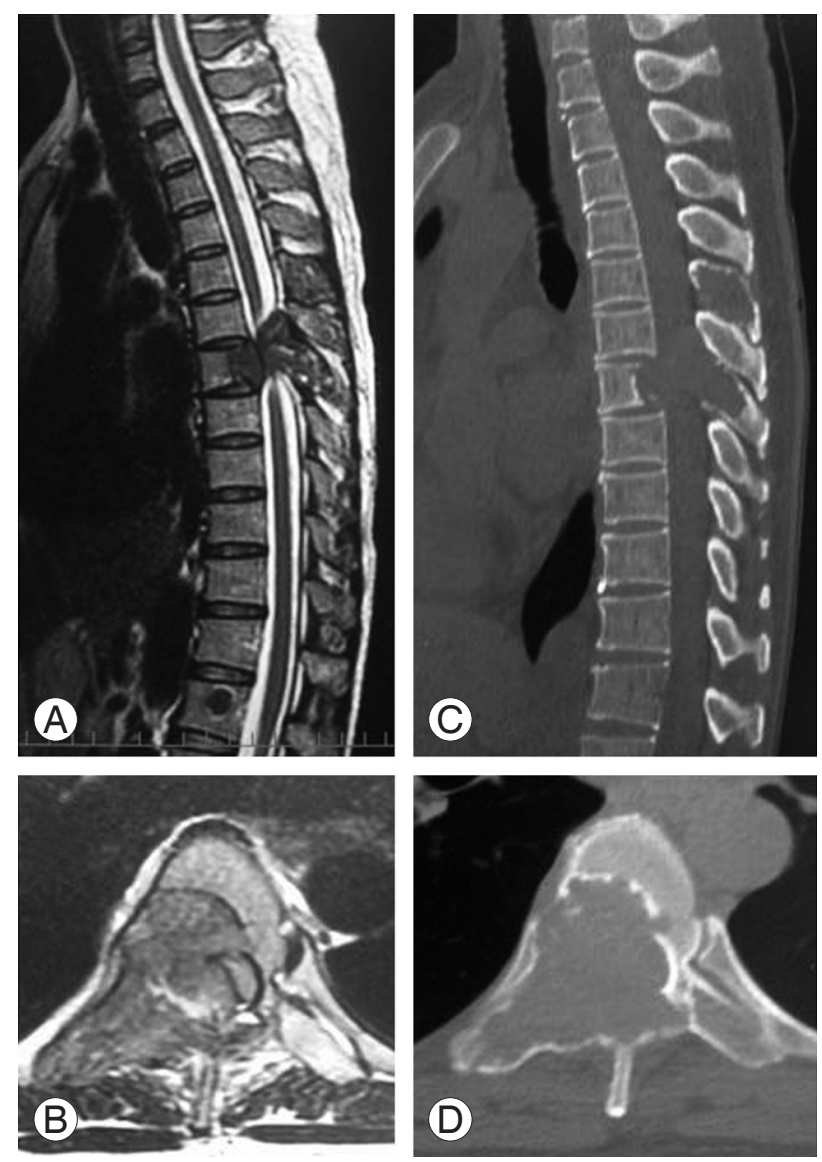

Fig. 2. T2 magnetic resonance images in the sagittal (A) and axial views (B) showing the tumor surrounding the spinal cord at the Th7 level. Sagittal (C) and axial (D) computed tomography reveal osteolysis at the Th7 level.
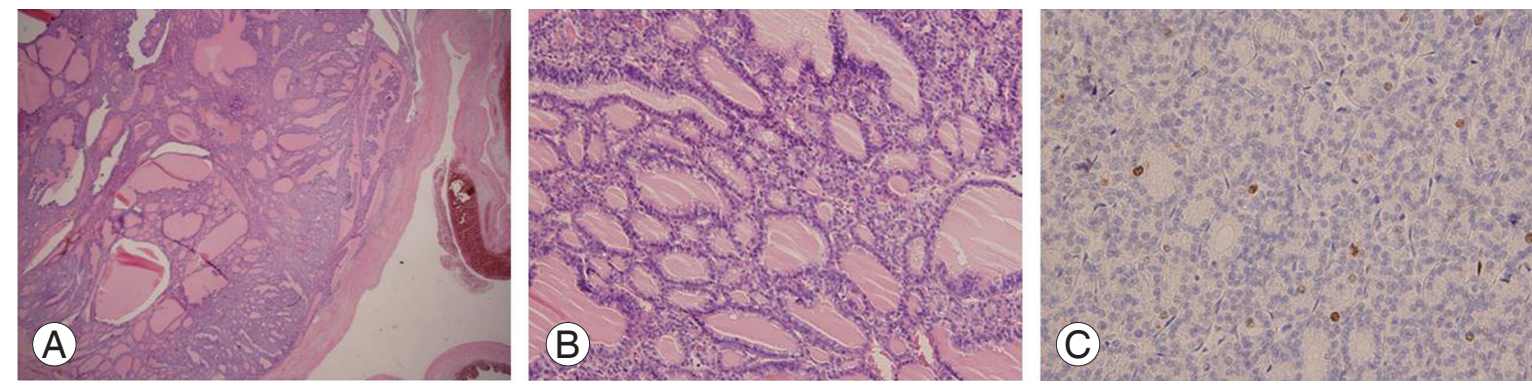

Fig. 1. (A) Pathological examination revealed proliferation of follicular epithelium in ovarian tissue. Thyroid tissue was also observed $(H \& E, \times 20)$. (B) Struma ovarii with features of a follicular tumor, with no malignancy (H\&E, $\times 100)$. (C) MIB-1 index of 1\%-2\% (Ki67, $\times 100)$. 


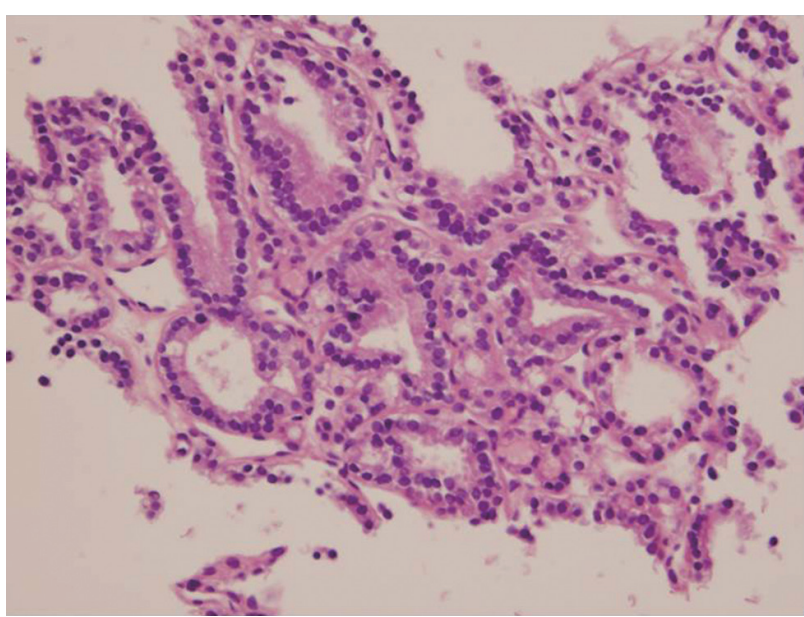

Fig. 3. Pathological examination revealed a metastatic follicular thyroid carcinoma in the Th7 lamina bone tissue. Atypical cells with nuclear swelling formed a follicle based on histological analysis $(H \& E, \times 400)$.

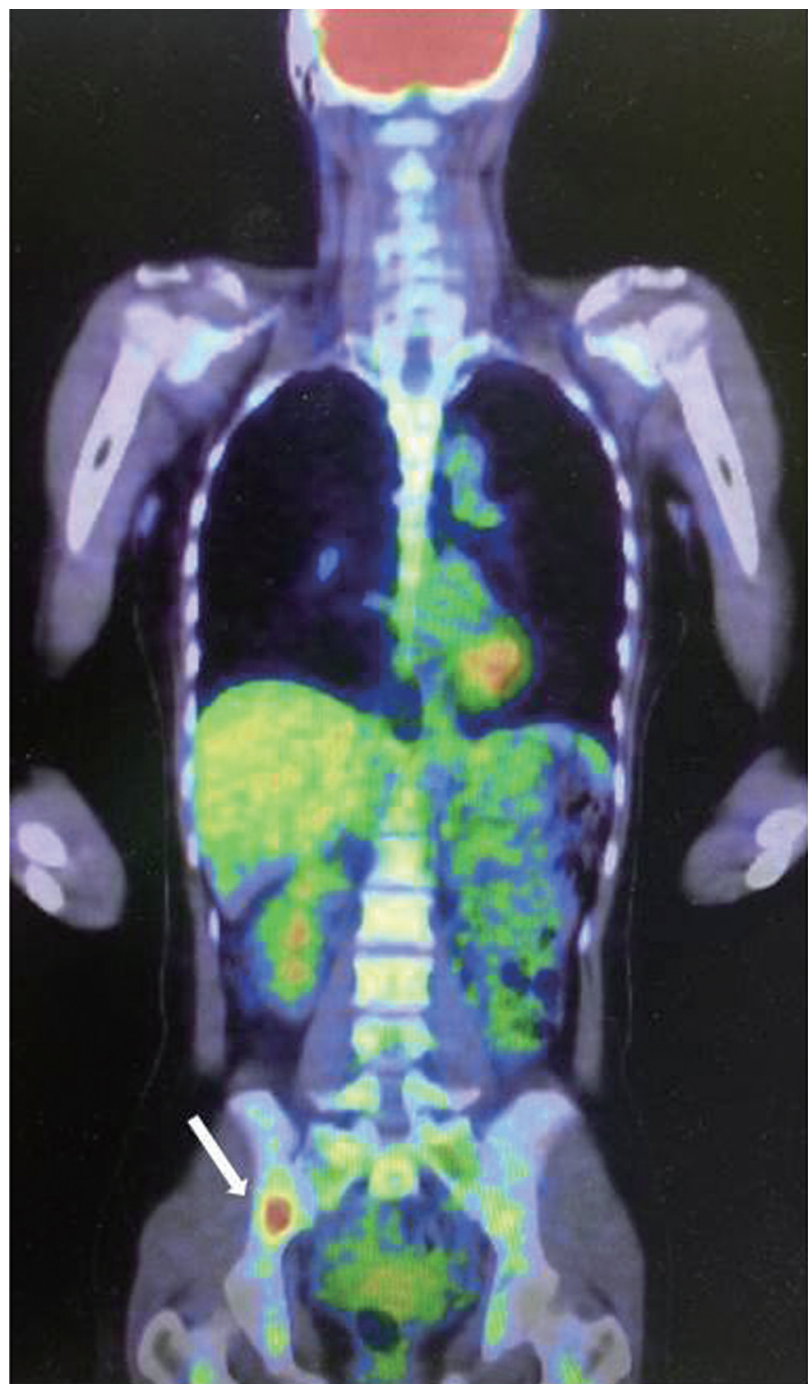

Fig. 4. Whole-body positron emission tomography revealed metastasis to the ilium (arrow) and no metastasis to the thyroid.
Th7 and lumbar brace. There was no worsening of neurological symptoms, and she was discharged. Currently, 9 months after treatment, there is no obvious tumor growth or muscle weakness, and she can walk unassisted.

\section{Discussion}

Struma ovarii is rare, constituting only $2.7 \%$ and $2 \%$ of all dermoid and ovarian tumors, respectively [2]. It is usually benign, and is histologically and pharmacologically identical to normal thyroid tissue. Malignant struma ovarii is very rare and occurs in $<1 \%$ of patients, and making a histological diagnosis is difficult [2]. Metastasis from malignant struma ovarii is also rare [3]. The disease can cause ascites and hydrothorax (Meigs' Syndrome), hyperthyroidism [6], or, rarely, symptoms from metastases [3]. Our patient exhibited elevated serum thyroglobulin, but did not have hyperthyroidism. In the first examination 10 years previously, the thyroid tissue in the lesion was histologically benign. Malignant struma ovarii was diagnosed because of spinal metastasis in the more recent examination. Previously, only 5 cases of struma ovarii with spinal metastasis have been reported (Table 1) [2,5-7].

Because of its rarity, the optimal treatment for malignant struma ovarii is unclear [4]. Previous cases have been treated with total abdominal hysterectomy and ovariectomy [8], and total thyroidectomy with subsequent Iodine-131 $\left({ }^{131} \mathrm{I}\right)$ radioablation [5]. Adjuvant therapy including chemotherapy and external beam radiation has been used for patients with advanced disease or recurrence [8]. As in thyroid cancer, recombinant human thyroid stimulating hormone has been used to increase the effectiveness of ${ }^{131}$ I radioablation [9]. Regarding spinal metastasis, Yamashita et al. [2] described a 32-yearold patient with a solitary L3 metastasis who underwent tumor resection and anterior spinal reconstruction. McDougall et al. [5] reported a 42-year-old patient with thoracic vertebral metastasis treated by tumor resection and fibular graft and two cycles of ${ }^{131}$ I radioablation. Chan and Farrell [6] described the case of a 27-year-old woman with persistent tracer uptake at T8 on a whole-body scan who was referred for radiotherapy. The postoperative courses in all three cases were uneventful. In the present case, the patient did not have a tumor in the thyroid and ovaries; therefore, ovariectomy and thyroidectomy were not performed. We were also concerned about temporary tumor enlargement that could result in paralysis after 
Table 1. Summary of cases of spinal metastasis of struma ovarii reported in the literature

\begin{tabular}{|c|c|c|c|c|}
\hline Study (year) & $\begin{array}{l}\text { Age of } \\
\text { patient } \\
\text { (yr) }\end{array}$ & $\begin{array}{l}\text { Level of } \\
\text { metastasis }\end{array}$ & $\begin{array}{l}\text { Time from struma } \\
\text { ovarii diagnosis to } \\
\text { spinal metastasis (yr) }\end{array}$ & Treatment \\
\hline \multirow[t]{2}{*}{ Dalgaard and Wetteland (1956) [7] } & 33 & Th10 & 4 & NA \\
\hline & 47 & Thoracolumbar & NA & NA \\
\hline McDougall et al. (1989) [5] & 42 & Th2 & 0 & $\begin{array}{l}\text { Tumor resection, fibular graft } \\
{ }^{131} \text { I Radioablation }\end{array}$ \\
\hline Chan and Farrell (2001) [6] & 27 & Th8 & 0.5 & Radiotherapy \\
\hline Yamashita et al. (2010) [2] & 32 & L3 & 0 & $\begin{array}{l}\text { Tumor resection, anterior } \\
\text { spinal reconstruction }\end{array}$ \\
\hline This case & 49 & Th7 & 10 & External beam radiotherapy \\
\hline
\end{tabular}

NA, not available; ${ }^{131}$ I, lodine-131.

${ }^{131}$ I radioablation, and so administered external beam radiation only. Although a considerable amount of three column of Th7 was involved, fortunately the patient complained of only back pain, without deterioration of neurological symptoms. This is probably due to slow progression, manifest as a recurrence after 10 years. As a result, there was no resulting increase in the tumor size and no muscle weakness in the legs thereafter.

The 10- and 25-year survival rates for benign lesions are $89 \%$ and $84 \%$, respectively [10]. Little is known about survival in malignant or metastatic cases because of their rarity. Some patients have a relatively good course even with distant metastases, while others die within a few years of onset [10]. These data emphasize the need for long-term follow-up of patients with malignant struma ovarii. It is also unclear which of the treatments described above is most effective in achieving higher survival and remission rates in patients with malignant struma ovarii.

The case reported here is the first example of malignant struma ovarii with thoracic metastasis that developed 10 years after resection. External beam radiation without tumor resection resulted in remission without worsening of symptoms to date. The case illustrates that metastasis from benign struma ovarii may occur after an extended disease-free period and that such cases require long-term follow-up.

\section{Conflict of Interest}

No potential conflict of interest relevant to this article was reported.

\section{References}

1. DeSimone CP, Lele SM, Modesitt SC. Malignant struma ovarii: a case report and analysis of cases reported in the literature with focus on survival and I131 therapy. Gynecol Oncol 2003;89:543-8.

2. Yamashita M, Ishii T, Ohtori S, et al. Metastasis of malignant struma ovarii to the lumbar spine. J Clin Neurosci 2010;17:269-72.

3. Yannopoulos D, Yannopoulos K, Ossowski R. Malignant struma ovarii. Pathol Annu 1976;11:403-13.

4. Steinman RA, De Castro IO, Shrayyef M, et al. Two cases of malignant struma ovarii with metastasis to pelvic bone. Gynecol Obstet Invest 2013;75:139-44.

5. McDougall IR, Krasne D, Hanbery JW, Collins JA. Metastatic malignant struma ovarii presenting as paraparesis from a spinal metastasis. J Nucl Med 1989;30:407-11.

6. Chan SW, Farrell KE. Metastatic thyroid carcinoma in the presence of struma ovarii. Med J Aust 2001; 175:373-4.

7. Dalgaard JB, Wetteland P. Struma ovarii; a follow-up study of 20 cases. Acta Chir Scand 1956;112:1-17.

8. Makani S, Kim W, Gaba AR. Struma Ovarii with a focus of papillary thyroid cancer: a case report and review of the literature. Gynecol Oncol 2004;94:8359.

9. Rotman-Pikielny P, Reynolds JC, Barker WC, Yen PM, Skarulis MC, Sarlis NJ. Recombinant human thyrotropin for the diagnosis and treatment of a highly functional metastatic struma ovarii. J Clin En- 
docrinol Metab 2000;85:237-44.

10. Robboy SJ, Shaco-Levy R, Peng RY, et al. Malignant struma ovarii: an analysis of 88 cases, including
27 with extraovarian spread. Int J Gynecol Pathol 2009;28:405-22. 